\title{
A Decline in Myometrial Nitric Oxide Synthase Expression Is Associated with Labor and Delivery
}

Raj K. Bansal, ${ }^{\star}$ Paul C. Goldsmith, ${ }^{\ddagger}$ Youping He, ${ }^{\S}$ Charles J. Zaloudek,, Jeffrey L. Ecker, ${ }^{\ddagger}$ and R. Kirk Riemer ${ }^{\ddagger}$

*Perinatal Associates, P.C., Portland, Oregon 97227-1654; ${ }^{*}$ Department of Obstetrics, Gynecology, and Reproductive Sciences, University of California, San Francisco, San Francisco, California 94143-0550; ${ }^{\S}$ Department of Surgery, Cardiothoracic Division, University of

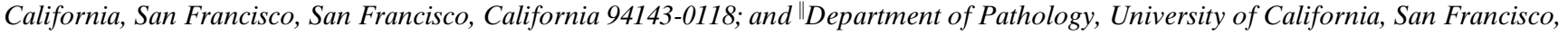
San Francisco, California 94143-0102

\begin{abstract}
The mechanisms that maintain relative uterine quiescence during pregnancy remain largely unknown. A possible role for nitric oxide has recently emerged, however, the expression of nitric oxide synthase within human myometrium at midgestation, a time when the uterus is normally quiescent, has not been investigated. The purpose of this study was to identify cell types in human myometrium that contain inducible nitric oxide synthase (iNOS), and to examine changes in its expression during pregnancy and labor. We found that iNOS is expressed in smooth muscle cells of pregnant myometrium. Expression of iNOS was highest in myometrium of preterm not-in-labor patients. At term, iNOS expression fell by $75 \%$, and was barely detectable in preterm in-labor or term in-labor specimens. There was no staining in the myocytes of nonpregnant myometrium. Western blotting also revealed a similar pattern of changes in iNOS expression. In summary, iNOS expression in the myocytes of human myometrium is increased greatly during pregnancy, and declines towards term or with labor. Significantly, preterm inlabor patients also had a large decline in iNOS expression. These data suggest that changes in myometrial iNOS expression may participate in the regulation of uterine activity during human pregnancy. (J. Clin. Invest. 1997. 99:25022508.) Key words: uterus $\bullet$ pregnancy maintenance $\bullet$ smooth muscle $\bullet$ immunohistochemistry $\bullet$ inducible nitric oxide synthase
\end{abstract}

\section{Introduction}

In pregnancy, the smooth muscle of the uterine myometrium undergoes important alterations that are critical to the uterus's role in providing a chamber in which the developing fetus can grow and mature. In the nonpregnant state, distention of the uterus leads to reflex contractions as it does in most smooth muscle. In normal pregnancies, however, the uterus distends

Address correspondence to Dr. R. Kirk Riemer, Department of Surgery, Cardiothoracic Division, University of California, San Francisco, 505 Parnassus Avenue, Box 0118, Room M593, San Francisco, CA 94143-0118. Phone: 415-476-2820; FAX: 415-476-9678; E-mail: riemer@socrates.ucsf.edu

Received for publication 21 October 1996 and accepted in revised form 27 February 1997.

J. Clin. Invest.

(C) The American Society for Clinical Investigation, Inc. 0021-9738/97/05/2502/07 \$2.00

Volume 99, Number 10, May 1997, 2502-2508 and grows to accommodate the enlarging conceptus, yet remains relatively quiescent until term. The consequences of a premature disruption in myometrial quiescence (preterm labor and delivery) are enormous, and constitute one of the leading causes of human perinatal morbidity and mortality (1). The mechanisms which enable the pregnant uterus to resist stretch-induced activation remain largely unknown, but probably reflect a complex balance of inhibitory and stimulatory factors. In the last few years a possible role for nitric oxide (NO), a potent inhibitor of smooth muscle contraction, has emerged.

NO, synthesized enzymatically by nitric oxide synthases (NOS), has been shown to have many important biological functions, including the regulation of vascular tone, regulation of cell-mediated cytotoxicity, and central and peripheral nervous system signal transmission (2). Three major isoforms of NOS have been identified (3-5), and can be grouped into two major classes: constitutive calcium-dependent, and inducible calcium-independent. The activities of the two constitutively expressed forms, endothelial constitutive NOS (ecNOS) and neuronal NOS (ncNOS), are regulated acutely by changes in intracellular calcium concentrations (6). The activity of the third isoform, inducible NOS (iNOS), is not controlled by changes in calcium concentration, but instead is transcriptionally regulated by a variety of growth factors and cytokines (7). The expression of iNOS can be induced in a wide variety of tissues and cultured cells, and the signaling molecules which cause this induction vary between responsive tissues (8). Notably, changes in iNOS expression have been reported in vascular smooth muscle in response to sepsis. In sepsis, increased expression of iNOS by vascular smooth muscle cells leads to vascular relaxation that is resistant to vasoconstrictors (9). Furthermore, there is a growing realization that cytokines and growth factors are produced in tissue-specific patterns in many physiologic states, so it is conceivable that iNOS may be upregulated in the uterine smooth muscle cells during pregnancy to assist in maintaining uterine quiescence.

The ability of exogenous NO to promote uterine relaxation is well documented. NO donors have been shown to cause uterine relaxation, and to inhibit contractions in vivo in humans. Nitroglycerine was shown to relax the uterus to facilitate extraction of retained placenta (10), and also, in an uncontrolled study, was considered to arrest preterm labor and to prolong gestation (11). Amyl nitrite was demonstrated to decrease the magnitude of uterine contractions induced by oxytocin, but not by spontaneous activity (12). NO donors have

1. Abbreviations used in this paper: ecNOS, endothelial constitutive nitric oxide synthase; iNOS, inducible nitric oxide synthase; ncNOS, neuronal constitutive nitric oxide synthase; NO, nitric oxide; TBS, Tris-buffered saline. 
also been shown to inhibit uterine contractions in pregnant rhesus monkeys (13) and sheep (14). Controlled in vitro studies have demonstrated similar inhibition in humans (15) and rats (16). Furthermore, studies have documented endogenous uterine production of NO during pregnancy. In a previous study from our laboratory, NOS activity increased in rat uteri during pregnancy and fell during labor at term; most of the change in activity was in the calcium-independent fraction, which likely represents iNOS (17). (Subsequent studies have verified that rat myometrial smooth muscle iNOS expression is upregulated in pregnancy and falls at term [18].) Upregulation of NOS activity in the uterus with pregnancy has also been demonstrated by others both in rats (19) and in rabbits (20), but it was not found in the guinea pig (21). In humans, there is evidence of endogenous NO production by the uterus in 24-h tissue cultures (15).

As of yet, however, upregulation of iNOS in human uterus has not been demonstrated, nor has the intrauterine site(s) of iNOS production during pregnancy been determined. It is unclear whether the increases in uterine NOS activity that have been observed are due to changes in myometrial or decidual NO production (20). Since NO has an estimated diffusion range of $100 \mu \mathrm{m}$ or less (22), we hypothesized that NO should be produced within the myometrium to suppress organized uterine contractions. In this study, we identified iNOS-containing cells in human myometrium in both the pregnant and nonpregnant state, and studied changes in iNOS expression with advancing gestation and with labor.

\section{Methods}

Five groups of patients were studied: $(a)$ nonpregnant, $(b)$ preterm not-in-labor, $(c)$ term not-in-labor, $(d)$ preterm in-labor, and $(e)$ term in-labor. Nonpregnant specimens were obtained from premenopausal women aged 41-48 yr undergoing hysterectomy for menometrorrhagia. Myometrium was sampled from the supraisthmic region of the uterus, taking care to avoid any endometrium or serosa. All pregnant specimens were obtained at the time of cesarean section from the myometrium of the upper edge of the hysterotomy incision. The pregnant patients (aged 18-38 yr) were grouped by both gestational age (term, 37-41 wk; preterm, 26-34 wk), and by whether or not active labor was present. All laboring patients had regular painful contractions with cervical dilation $>3 \mathrm{~cm}$. All nonlaboring patients had neither cervical change nor perceptible regular contractions. Term patients had cesarean sections because of cephalo-pelvic disproportion, prior uterine incisions, fetal malformations, and breech presentation. Preterm in-labor patients had cesareans for breech presentation and placenta previa. Preterm not-in-labor patients had cesareans for fetal distress, breech presentation, placenta previa, cervical cancer, and maternal pheochromocytoma. The study was approved by the Committee for Human Research of the University of California, San Francisco, and patients were given appropriate informed consent.

Immunohistochemistry. After collection, a cube of each tissue specimen, approximately $5 \times 5 \times 5 \mathrm{~mm}$ and grossly devoid of decidua or serosa, was fixed by immersion in a solution containing (final concentrations) $4 \%$ formaldehyde, $0.1 \mathrm{M}$ phosphate buffer ( $\mathrm{pH} 7.6)$, and $50 \%$ methanol for at least $24 \mathrm{~h}$ at $4^{\circ} \mathrm{C}$. The specimens were paraffin-embedded, sectioned at $8 \mu \mathrm{m}$, and collected on saline-coated glass slides. Sections were then deparaffinized in xylene, rehydrated through graded alcohols, and equilibrated in $0.05 \mathrm{M}$ Tris-buffered saline (TBS), $\mathrm{pH}$ 7.4. Endogenous peroxidase activity was quenched in $2 \% \mathrm{H}_{2} \mathrm{O}_{2}$ in $60 \%$ methanol; the tissue was permeabilized with $0.2 \%$ Triton X-100 in TBS, and nonspecific binding was blocked with $2 \%$ normal goat serum in TBS (blocking solution).

Immunostaining was conducted with a rabbit polyclonal antibody specific for iNOS (No. N32030; Transduction Labs, Lexington, KY) which was provided as an $\operatorname{IgG}$ fraction affinity-purified against the peptide immunogen. The antiserum was diluted to $5 \mu \mathrm{g} / \mathrm{ml}$ in blocking solution, and was incubated on sections overnight at $4^{\circ} \mathrm{C}$. After washing in TBS, sections were exposed to two sequential cycles of 1:100 goat anti-rabbit $\mathrm{IgG}$, and 1:100 rabbit peroxidase-antiperoxidase complex $(20 \mathrm{mg} / \mathrm{ml}$ stock concentration; Cappel-Organon Teknika, Durham, NC) in blocking solution. After thorough rinsing, sections were reacted in substrate consisting of $0.5 \mathrm{mg}$ diaminobenzidine $(\mathrm{DAB}) / \mathrm{ml}$ of $1 \% \mathrm{H}_{2} \mathrm{O}_{2}$ in $0.1 \mathrm{M}$ Tris- $\mathrm{HCl}$ for $3 \mathrm{~min}$. Immunoreactive iNOS was identified by the formation of a brown precipitate from this reaction. Samples from all five groups of patients were tested in each experiment to avoid misinterpretation of interexperimental variation in staining intensities as differences between the groups. Immunostaining controls routinely performed on each sample involved incubation in blocking solution overnight in place of the primary antibody. The specificity of the antisera was verified by substitution of the same concentration of normal rabbit IgG as was used for the antiiNOS IgG, which produced no discernible staining (results not shown). The lack of cross-reactivity of this antiserum with ecNOS was confirmed by the absence of staining of vascular endothelium in the myometrial sections and by Western blotting. The lack of cross-reactivity with ncNOS was confirmed by Western blotting since ncNOS was not detectable in the myometrial samples that were positive for iNOS. Also, the specificity of the immunostaining for iNOS was verified in a few samples using a different iNOS antisera, a mouse monoclonal antibody to iNOS (No. N32020; Transduction Labs) that is affinity-purified against the immunogen, and has been previously reported to be specific for iNOS in humans (23). Sections were incubated with this antibody overnight at $4^{\circ} \mathrm{C}$ at $2.5 \mu \mathrm{g} / \mathrm{ml}$ diluted in $2 \%$ normal horse serum in TBS. Sections were then reacted with an avidin-biotin peroxidase system for mouse IgG (Vectastain ABC Elite Kit; Vector Laboratories, Inc., Burlingame, CA) according to the manufacturer's instructions. Staining was completed using the same $\mathrm{DAB}$ formulation as above for $5 \mathrm{~min}$.

In all samples, separate sections were stained with Gill's hematoxylin No. 2 and eosin to confirm histological structures. To aid in the identification of DAB immunoreactive cells, some sections were lightly counterstained with Gill's hematoxylin No. 2 or toluidine blue.

Although qualitative differences in iNOS staining were readily apparent, cells containing iNOS were quantified morphometrically. Immunostained sections without counterstain were examined under high power $(\times 312)$ with a light microscope fitted with a Weibel reticule. For each specimen, the proportion of immunostained cells in five randomly selected fields was determined and expressed as a percentage. Significant differences between groups were assessed by analysis of variance (StatView; Abacus Concepts Inc., Berkeley, CA).

Western blotting. Myometrial protein extracts were prepared by homogenizing tissue in $5 \mathrm{vol}(1 \mathrm{ml} / \mathrm{g}$ wet wt) of boiling $10 \mathrm{mM}$ Tris$\mathrm{HCl}(\mathrm{pH} 7.4)$ containing $1 \%$ SDS, heating the homogenate in a microwave oven for $10 \mathrm{~s}$, and then removing insoluble debris by centrifugation at $7,500 \times g$ for $15 \mathrm{~min}$ at $15^{\circ} \mathrm{C}$. Protein concentration was determined using protein reagent (Bio-Rad Laboratories, Richmond, CA) and bovine serum albumin reference standard. Electrophoresis was performed using $100-\mu \mathrm{g}$ aliquots of protein extract in $1 \times$ loading buffer (final concentrations: $12.5 \mathrm{mM}$ Tris- $\mathrm{HCl}, \mathrm{pH}$ 6.8, 0.4\% SDS, $2 \%$ glycerol, $1 \%$ 2-mercaptoethanol, and $0.5 \%$ bromphenol blue) in a $6 \%$ SDS-PAGE gel.

Western blotting was performed after electrophoretic transfer of resolved proteins onto Immobilon-P paper (Millipore Corp., Bedford, MA). An affinity-purified (against the peptide immunogen) rabbit polyclonal antibody to human iNOS (No. SC-649; Santa Cruz Biotechnology, Santa Cruz, CA) was used at a concentration of $2 \mu \mathrm{g} / \mathrm{ml}$. Peroxidase-conjugated donkey anti-rabbit secondary antibody (Amersham Corp., Arlington Heights, IL) was detected (Supersignal CLHRP Substrate System; Pierce, Rockford, IL). Specificity of the antisera was assessed by use of positive and negative control antigens that confirmed selectivity for human iNOS, the lack of reactivity with ei- 

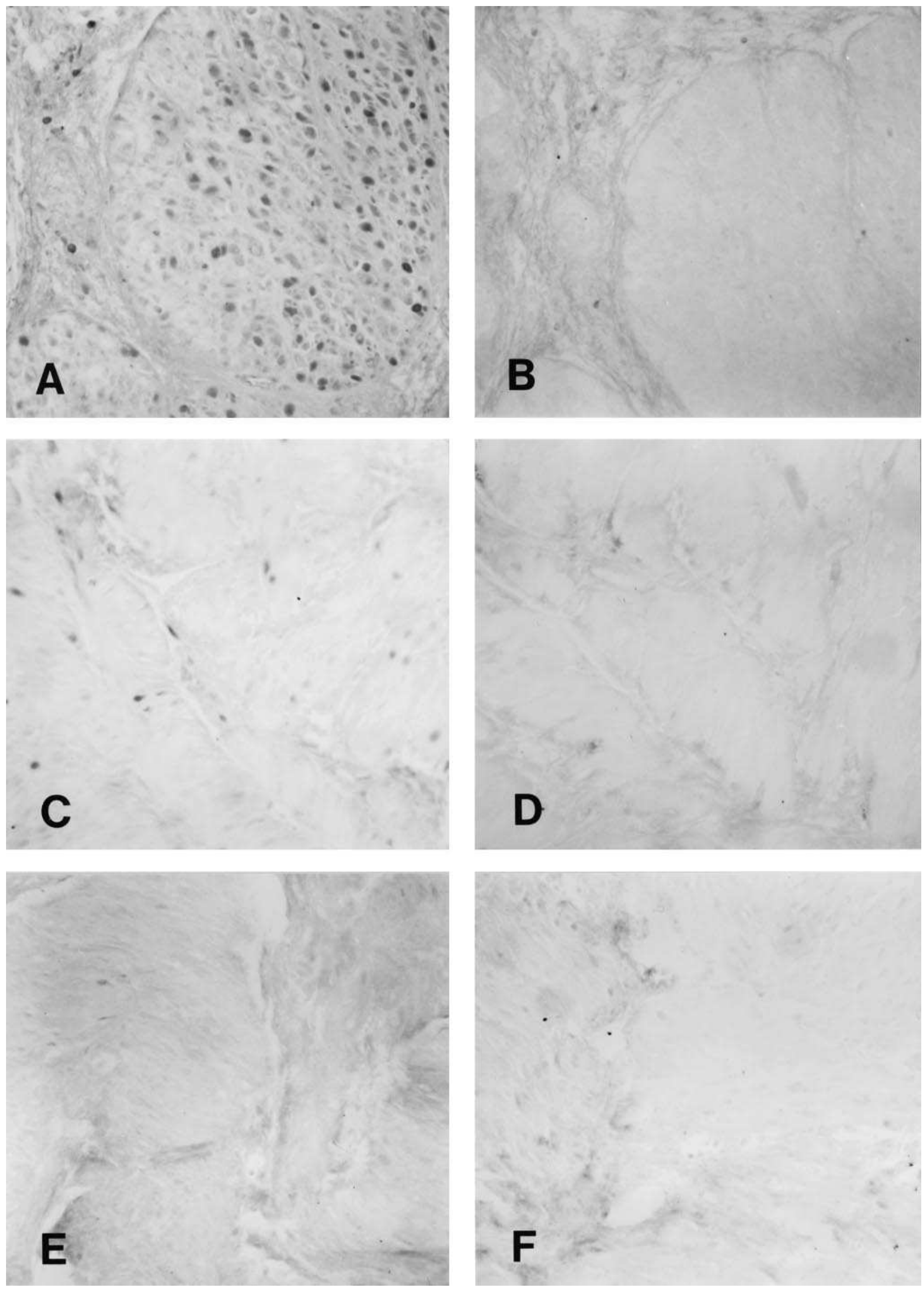
ther murine iNOS or other NOS isoforms, and the lack of the signal from preimmune serum (data not shown). Protein extracts from DLD-1 human colorectal carcinoma cells induced to express iNOS with cytokines (24) served as the positive control for human iNOS. Quantitation of the autoradiographic signals was performed using a scanning densitometer, and the relative optical density of the iNOS band for equal areas of several specimens from each group was compared by analysis of variance. Data are expressed as means \pm SE.

\section{Results}

Hematoxylin and eosin staining confirmed the presence of normal-appearing myometrium without decidua or serosa in all tissue samples. As expected, the myocytes within the pregnant uteri had undergone hypertrophy, and the tissue was more edematous when compared with the nonpregnant uterine samples.

In the pregnant uteri, immunoreactive iNOS was localized exclusively in myocytes. Expression of iNOS was greatest in preterm not-in-labor samples $(n=5)$, in which $8.2 \pm 1.1 \%$ of the myocytes contained iNOS (Fig. $1 A$ ). The iNOS-containing myocytes were dispersed diffusely throughout the myometrium without any obvious pattern, and did not appear histologically different from neighboring, unstained myocytes. Staining within individual cells varied in intensity, but was mostly strong and uniform. Adjacent sections lightly counterstained with hematoxylin confirmed that the staining was in the extranuclear cytoplasm. Control sections without primary antibody (Fig. $1 B$ ), or where normal rabbit IgG was substituted for the primary antisera, showed no specific staining; this was true for all of the pregnant and nonpregnant uterine sample controls. Samples immunostained with the monoclonal antibody revealed similar staining, confirming the iNOS immunoreactivity.

Term not-in-labor samples $(n=9)$ had significantly fewer iNOS-positive myocytes $(2.1 \pm 0.7 \%)$, and reduced staining intensity within individual cells (Figs. $1, C$ and $D$ ). This group of specimens was also heterogeneous; some specimens displayed almost no staining, while some had proportions of iNOS-containing myocytes that approached the preterm not-in-labor specimens. The intensity of staining within individual cells in all of the term not-in-labor samples, however, was qualitatively less than that in the preterm not-in-labor samples. The differences within patient groups could not be correlated with any obvious clinical differences in the patients, including gestational age, medication use, or indication for cesarean section.

Samples from the two laboring groups, preterm $(n=3)$ and term $(n=5)$, had little or no detectable iNOS expression $(0.5 \pm 0.5 \%$ and $0.6 \pm 0.4 \%$, respectively). Two samples from the preterm group and one sample from the term group had no evidence of specific immunostaining. The rest of the samples in both groups had only occasional myocytes that weakly immunostained for iNOS (Fig. $1 E$ and $F$ ).

The large qualitative differences observed in iNOS immunostaining between the groups were confirmed by a quantitative analysis of the staining (Fig. 2). There was a significant difference in the percentage of iNOS-containing myocytes between the four groups of pregnant myometrial samples $(P<$

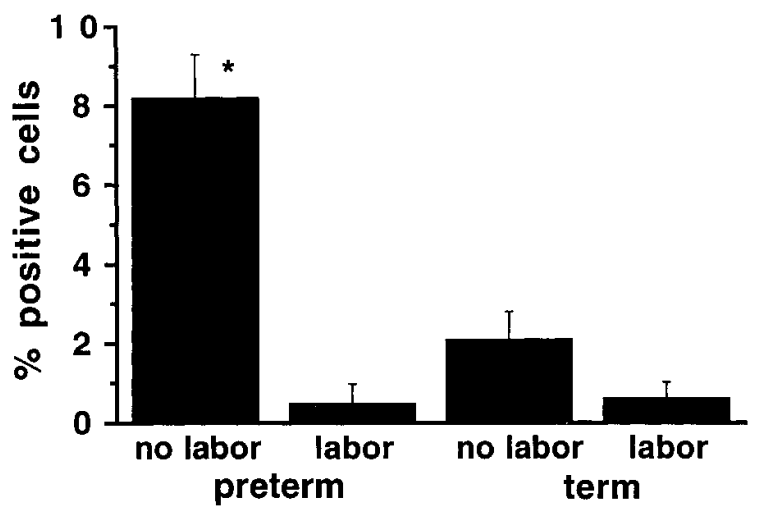

Figure 2. Percentage of myocytes containing iNOS within each group of myometrial biopsies during pregnancy (mean $\pm \mathrm{SE}$ ). The numbers of patients within each group were as follows: preterm no labor, 5; preterm labor, 3; term no labor, 9; term labor, 5. *Significantly greater than each of the other three groups $(P<0.0001)$.

0.0001). Furthermore, pairwise posthoc testing (Fisher's protected least significant difference) revealed that staining in the preterm not-in-labor group was significantly greater than that in the term not-in-labor group $(P<0.0001)$, the preterm inlabor group $(P<0.0001)$, and the term in-labor group $(P<$ $0.0001)$. The staining in the term not-in-labor group was greater than in both laboring groups, but this was not statistically significant.

In contrast to the pregnant uterine samples, the nonpregnant uterine samples $(n=4)$ had no detectable myocyte staining. The only immunostaining observed was in occasional cells in the connective tissue surrounding blood vessels; these cells histologically appeared to be mast cells (Fig. 3). The staining was somewhat granular, asymmetric, and cytoplasmic in location. Histochemical staining of adjacent sections with toluidine blue revealed metachromatic purple granules in the cytoplasm of these cells, which confirms that they are mast cells. Control sections without primary antibody had no specific immunostaining.

Western blotting of myometrial protein extracts revealed findings similar to those obtained by immunohistochemistry. Although equivalent amounts of protein were loaded for each sample, only preterm not-in-labor extracts contained significant amounts of iNOS (Fig. 4). Quantitation of the relative iNOS band intensity (Fig. 5) revealed a significant difference between the groups $(P=0.01)$. Pairwise posthoc testing confirmed that the preterm not-in-labor extracts contained significantly greater iNOS protein than the other four groups $(P<$ 0.01 for all).

\section{Discussion}

This study demonstrated the presence of iNOS protein in human myometrium. Whereas in the nonpregnant state immunoreactive iNOS was found only in tissue mast cells, during

Figure 1. Immunohistochemical localization of iNOS-containing cells in human myometrium during pregnancy. $(A)$ Preterm not-in-labor $(\times 175) ;(B)$ preterm not-in-labor control $(\times 175) ;(C)$ term not-in-labor $(\times 175) ;(D)$ term not in labor control $(\times 175) ;(E)$ term in-labor $(\times 175)$; $(F)$ preterm in-labor $(\times 175)$. Immunostaining in control sections involved incubation in buffer in place of the primary antiserum. 


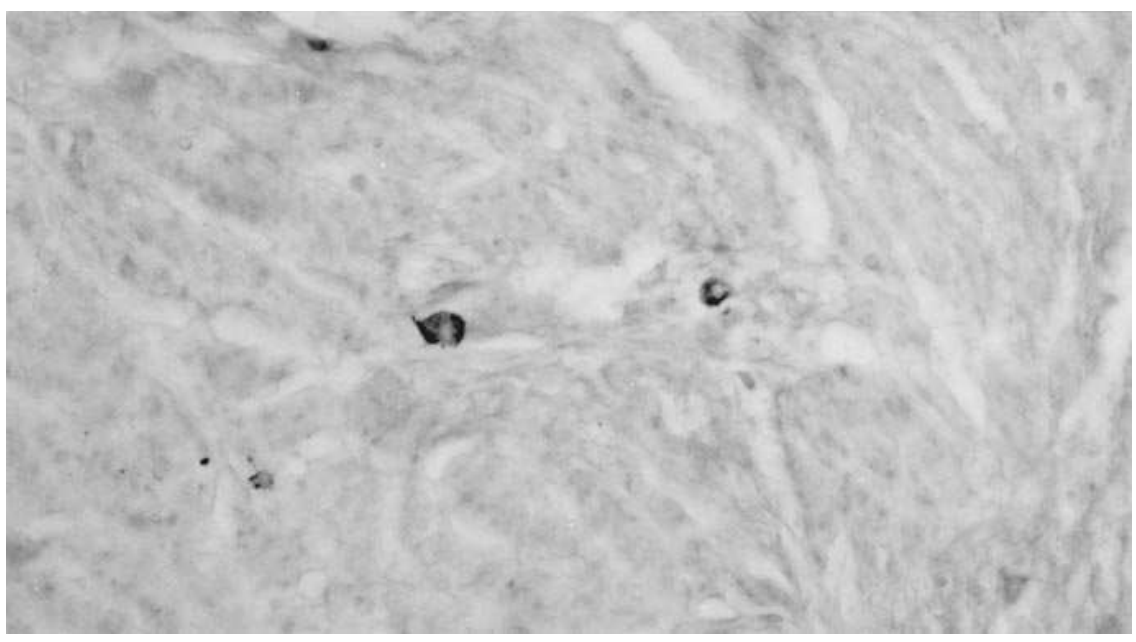

Figure 3. Immunohistochemical localization of iNOS-containing cells in nonpregnant human myometrium $(\times 412)$. Labeled cells were identified as mast cells by toluidine blue staining of adjacent sections. There was no myocyte staining detected. pregnancy iNOS expression increased greatly and appeared predominantly in myocytes. Myocyte iNOS expression was present in specimens from preterm, quiescent uteri. As gestation progressed toward term, iNOS expression decreased, and was nearly absent with the commencement of labor. It is important to note that a precipitous decline in iNOS expression also occurred in the preterm in-labor myometrium. Similar results were obtained using both immunohistochemistry and Western blotting. These findings support the hypothesis that induction of iNOS expression in myocytes during pregnancy occurs along a time course consistent with a role for NO in the maintenance of appropriate uterine quiescence.

Prior studies have indicated several possible sites of NO production in the uterus. NADPH diaphorase staining (which identifies all isoforms of NOS and several other enzymes [25]) in the pregnant rat uterus identified potential NOS in decidua, blood vessels, nerves, and muscle bundles $(17,26)$. Previous studies showing increased NOS activity during pregnancy used full-thickness uterine tissues which presumably included decidua $(17,19)$. Thus, it was not clear which isoforms of NOS were responsible for the increased activity, or in which cell types or tissues it was induced. While rabbit decidua did show increased NOS activity during pregnancy (20), given NOs limited range of diffusion, it seems unlikely that decidual NO has much effect on myometrial contractility; this is especially true

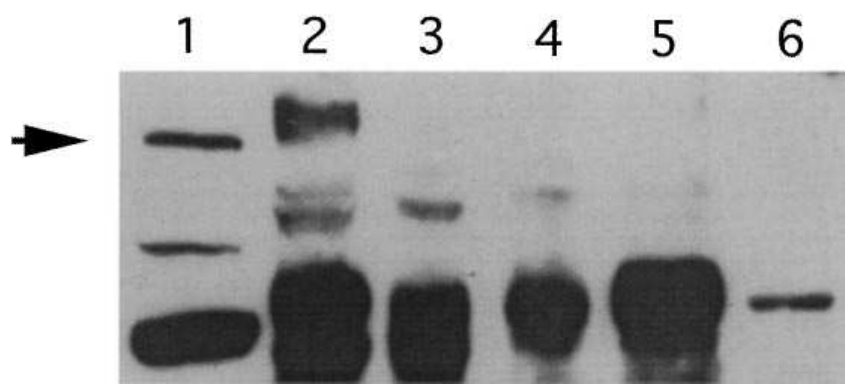

Figure 4. Western blotting for iNOS in human myometrial protein extracts $(100 \mu \mathrm{g}$ of total protein in each lane). The arrow marks putative iNOS band at $\sim 130 \mathrm{kD}$. Lane 1, positive control; lane 2, preterm not-in-labor; lane 3, preterm in-labor; lane 4, term not-in-labor; lane 5 , term in-labor; lane 6 , nonpregnant. for humans, where the myometrium is $\sim 20$ times thicker than in the rabbit. Importantly, this report reveals enhanced iNOS expression within the myometrium, where NO production is more likely to be directly related to the regulation of myometrial tone.

In the nonpregnant myometrium, there were two findings of note: the lack of myocyte immunostaining for iNOS, and the occasional iNOS-containing mast cells. The function of these myometrial mast cells is presumably immunologic, since their minimal numbers and localization around blood vessels make it unlikely that they would have a major effect on myometrial contractility. An immunohistochemical study using nonpregnant mice also localized myometrial iNOS predominantly in mast cells (27). These investigators, however, have reported that there was some immunostaining within macrophages. The lack of macrophage staining in the present study may be due to species differences, since it is controversial whether human macrophages contain iNOS (28). Our results are supported by another recent study which reported that NADPH diaphorase

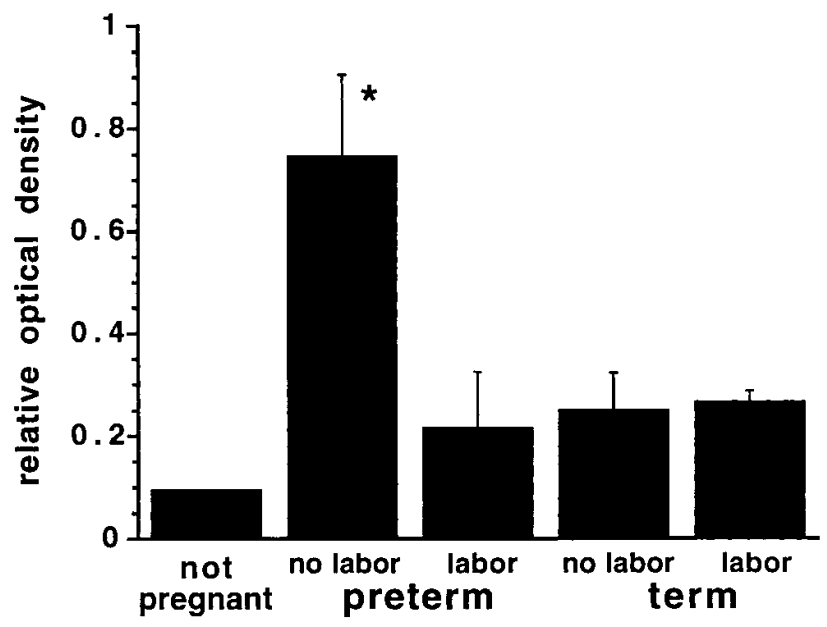

Figure 5. Relative optical density (mean \pm SE) of iNOS band in autoradiographs of Western blots for each group of myometrial protein extracts. All groups included extracts from three patients except the nonpregnant group, which had two patients. *Significantly greater than each of the other four groups $(P<0.01)$. 
staining for NOS activity in the nonpregnant human uterus revealed no myocyte NOS (29). (Also, immunolocalization studies from our laboratory have demonstrated that myometrial smooth muscle iNOS expression is not detectable in randomcycling rats, but is increased in pregnancy [18].)

The compelling finding in this study was the marked induction of iNOS expression within human myocytes during preterm pregnancy, and the decline in iNOS expression near term and with labor. This finding supports the concept that NO may be a significant regulator of myometrial tone during gestation. Using human myometrium in a muscle bath preparation, another group of investigators has shown that stimulating endogenous NOS activity abolished spontaneous contractions (30), and that pregnant myometrium was more sensitive to NO than was nonpregnant myometrium (15). This group also estimated endogenous myometrial NOS activity by measuring nitrite/nitrate generation in 24-h tissue cultures, and concluded that the activity was not different among their three study groups: $(a)$ pregnant not-in-labor, $(b)$ pregnant in-labor, $(c)$ and nonpregnant (15). Nearly all the pregnant patients in that study, however, were at term, and therefore their results may be consistent with our study. Similarly, a report of NOS activity in human myometrium (31) using the arginine-to-citrulline conversion assay (which found no difference in activity between nonlabored and labored uteri) also examined specimens from only term uteri, and is therefore consistent with our findings.

Since myocyte iNOS expression increases with pregnancy, what factors are responsible for this induction? Several cytokines (IL-1, TNF- $\alpha$, colony stimulating factor-1 [CSF-1], and TGF- $\beta$ ) have been shown to increase in the uterus during pregnancy (32-34), and ovarian hormones may also induce iNOS expression in the uterus (27). Preliminary studies performed in our lab show that M-CSF-1 and TGF- $\beta$ together induce iNOS in cultured mouse uterine myocytes (our unpublished data). The hormones that might reduce iNOS expression at term are not known. The recently reported decline in human myometrial progesterone receptor concentration in laboring women (35), however, would provide a mechanism for reduced progesterone-driven cytokine production as a potential cause of the reduced iNOS reduction in laboring uteri. Future studies may be able to further resolve which signaling molecules regulate iNOS expression during human pregnancy.

The pattern of myocyte iNOS expression that was found in the preterm not-in-labor samples was unusual in that only a minor proportion of cells expressed this enzyme at high levels, yet immunostaining for alpha smooth muscle actin (not shown) confirmed that the myometrium is composed of an otherwise homogenous population of myocytes. Perhaps myocyte NO functions to prevent the coordinated uterine contractions required for labor rather than to completely suppress all uterine activity. Some uterine tone is maintained during gestation, and scattered myocytes producing NO may act to prevent large organized contractions. The observed pattern of localized iNOS expression suggests a specialization of a subset of myocytes which may not be limited to the expression of iNOS. For example, a markedly similar pattern of expression was reported recently for the human myometrial oxytocin receptor (36).

\section{Acknowledgments}

We thank Evelyn N. Garrett for her excellent technical assistance. This work was supported by the March of Dimes grant FY95-0319
(R.K. Riemer) and National Institutes of Health grant HD26152 (R.K. Riemer).

\section{References}

1. Creasy, R. 1994. Preterm labor and delivery. In Maternal-Fetal Medicine: Principles and Practice. R. Creasy and R. Resnik, editors. W.B. Saunders, Philadelphia. 494-495.

2. Lowenstein, C.J., J.L. Dinerman, and S.H. Snyder. 1994. Nitric oxide: a physiologic messenger. Ann. Intern. Med. 120:227-237.

3. Bredt, D.S., P.M. Hwang, C.E. Glatt, C. Lowenstein, R.R. Reed, and S.H. Snyder. 1991. Cloned and expressed nitric oxide synthase structurally resembles cytochrome P-450 reductase. Nature (Lond.). 351:714-718.

4. Lamas, S., P.A. Marsden, G.K. Li, P. Tempst, and T. Michel. 1992. Endothelial nitric oxide synthase: molecular cloning and characterization of a distinct constitutive enzyme isoform. Proc. Natl. Acad. Sci. USA. 89:6348-6352.

5. Xie, Q.W., H.J. Cho, J. Calaycay, R.A. Mumford, K.M. Swiderek, T.D. Lee, A. Ding, T. Troso, and C. Nathan. 1992. Cloning and characterization of inducible NOS from mouse macrophages. Science (Wash. DC). 256:225-228.

6. Forstermann, U., J.S. Pollock, H.W. Schmidt, M. Heller, and F. Murad 1991. Calmodulin-dependent endothelium-derived relaxing factor/nitric oxide synthase activity is present in the particulate and cytosolic fractions of bovine aortic endothelial cells. Proc. Natl. Acad. Sci. USA. 88:1788-1792.

7. Ding, A.H., C.F. Nathan, and D.L. Stuejr. 1988. Release of reactive nitrogen intermediates and reactive oxygen intermediates from mouse peritoneal macrophages. J. Immunol. 141:2407-2412.

8. Nathan, C., and Q.W. Xie. 1994. Regulation of biosynthesis of nitric oxide. J. Biol. Chem. 269:13725-13728.

9. Moncada, S., and A. Higgs. 1993. The L-arginine-nitric oxide pathway. $N$. Engl. J. Med. 329:2002-2012.

10. Peng, A.T., R.S. Gorman, S.M. Shulman, E. DeMarchis, K. Nyunt, and L.S. Blancato. 1989. Intravenous nitroglycerin for uterine relaxation in the postpartum patient with retained placenta [letter]. Anesthesiology. 71:172-173.

11. Lees, C., S. Campbell, E. Jauniax, R. Brown, B. Ramsay, D. Gibb, S. Moncada, and J.F. Martin. 1994. Arrest of preterm labor and prolongation of gestation with glyceryl trinitrate, a nitric oxide donor. Lancet. 343:1325-1326.

12. Kumar, D., P.A. Zourlas, and A.C. Barnes. 1965. In vivo effect of amyl nitrite on human pregnant uterine contractility. Am. J. Obstet. Gynecol. 91: 1066-1068.

13. Jennings, R.W., T.E. MacGillivray, and M.R. Harrison. 1993. Nitric oxide ablates preterm labor in the rhesus monkey. J. Matern. Fetal Med. 2:170-175.

14. Heymann, M.A., B. Bootstaylor, C. Roman, E.S. Natuzzi, J.T. Parer, M.R. Harrison, and R.K. Riemer. 1994. Glyceryl trinitrate stops active labour in sheep. In The Biology of Nitric Oxide. Vol. 3. S. Moncada, M. Feelisch, R. Busse, and E.A. Higgs, editors. Portland Press, London. 201-203.

15. Buhimschi, I. C. Yallampalli, Y.L. Dong, and R.E. Garfield. 1995. Involvement of a nitric oxide-cyclic guanosine monophosphate pathway in control of human uterine contractility during pregnancy. Am. J. Obstet. Gynecol. 172:1577-1584.

16. Yallampalli, C., R.E. Garfield, and M. Byam-Smith. 1993. Nitric oxide inhibits uterine contractility during pregnancy but not during delivery. Endocrinology. 133:1899-1902.

17. Natuzzi, E.S., P. Ursell, C. Buscher, M. Harrison, and R.K. Riemer. 1993. Nitric oxide synthase activity in the pregnant uterus decreases at parturition. Biochem. Biophys. Res. Commun. 194:1-8.

18. Riemer, R.K., C. Buscher, R.K. Bansal, S.M. Black, Y. He, and E.S Natuzzi. 1997. Increased expression of nitric oxide synthase in the myometrium of the pregnant rat uterus. Am. J. Physiol. In press.

19. Yallampalli, C., M. Byam-Smith, S.O. Nelson, and R.E. Garfield. 1994. Steroid hormones modulate the production of nitric oxide and cGMP in the rat uterus. Endocrinology. 134:1971-1974.

20. Sladek, S.M., A.C. Regenstein, D. Lykins, and J.M. Roberts. 1993. Nitric oxide synthase activity in the pregnant rabbit uterus decreases on the last day of pregnancy. Am. J. Obstet. Gynecol. 169:1285-1291.

21. Weiner, C.P., I. Lizasoain, S.A. Baylis, R.G. Knowles, I.G. Charles, and S. Moncada. 1994. Induction of calcium-dependent nitric oxide synthases by sex hormones. Proc. Natl. Acad. Sci. USA. 91:5212-5216.

22. Malinski, T., Z. Tahada, S. Grunfeld, S. Patton, M. Kapturczak, and P. Tomoulian. 1993. Diffusion of nitric oxide in the aorta wall monitored in situ by porphyrinic microsensors. Biochem. Biophys. Res. Commun. 193:1076-1082.

23. Rowe, A., A. Farrell, S.H. Kazmi, and C.B. Bunker. 1994. Expression of inducible nitric oxide synthase in dermal microvasculature in psoriasis. Lancet. 344:1371.

24. Sherman, P.A., V.E. Laubach, B.R. Reep, and E.R. Wood. 1993. Purification and cDNA sequence of an inducible nitric oxide synthase from a human tumor cell line. Biochemistry. 32:11600-11605.

25. Tracey, W.R., M. Nakane, J.S. Pollock, and U. Forstermann. 1993. Nitric oxide synthases in neuronal cells, macrophages and endothelium are NADPH diaphorases, but represent only a fraction of total cellular NADPH diaphorase activity. Biochem. Biophys. Res. Commun. 195:1035-1040.

26. Yallampalli, C., H. Izumi, M. Byam-Smith, and R.E. Garfield. 1994. An 
L-arginine-nitric oxide-cyclic guanosine monophosphate system exists in the uterus and inhibits contractility during pregnancy. Am. J. Obstet. Gynecol. 170: $175-185$.

27. Huang, J., K.F. Roby, J.L. Pace, S.W. Russell, and J.S. Hunt. 1995. Cellular localization and hormonal regulation of inducible nitric oxide synthase in cycling mouse uterus. J. Leukocyte Biol. 57:27-35.

28. Denis, M. 1994. Human monocytes/macrophages: NO or no NO? J. Leukocyte Biol. 55:682-684.

29. Telfer, J.F., F. Lyall, J.E. Norman, and I.T. Cameron. 1995. Identification of nitric oxide synthase in human uterus. Hum. Reprod. 10:19-23.

30. Izumi, H., C. Yallampalli, and R.E. Garfield. 1993. Gestational changes in L-arginine-induced relaxation of pregnant rat and human myometrial smooth muscle. Am. J. Obstet. Gynecol. 169:1327-1337.

31. Ramsay, B., S.R. Sooranna, and M.R. Johnson. 1996. Nitric oxide synthase activities in human myometrium and villous trophoblast throughout pregnancy. Obstet. Gynecol. 87:249-253.

32. Bry, K., and M. Hallman. 1993. TGF-beta 2 prevents preterm delivery induced by IL-1 alpha and TNF-alpha in the rabbit. Am. J. Obstet. Gynecol. 168:1318-1322.

33. Das, S.K., K.C. Flanders, G.K. Andrews, and S.K. Dey. 1992. Expression of transforming growth factor-beta isoforms (beta 2 and beta 3 ) in the mouse uterus: analysis of the periimplantation period and effects of ovarian steroids. Endocrinology. 130:3459-3466.

34. Arceci, R.J., F. Shanahan, E.R. Stanely, and J.W. Pollard. 1989. Temporal expression and localization of colony-stimulating factor 1 (CSF-1) and its receptor in the female reproductive tract are consistent with CSF-1 regulated placental development. Proc. Natl. Acad. Sci. USA. 86:8818-8822.

35. How, H., Z.H., Huang, J. Zuo, Z.M. Lei, J.A. Spinnato, 2nd, and C.V. Rao. 1995. Myometrial estradiol and progesterone receptor changes in preterm and term pregnancies. Obstet. Gynecol. 86:936-940.

36. Kimura, T., M. Takemura, S. Nomura, T. Nobunaga, Y. Kubota, T. Inoue, K. Hashimoto, I. Kumazawa, Y. Ito, K. Ohashi, et al. 1996. Expression of oxytocin receptor in human pregnant myometrium. Endocrinology. 137:780-785. 